\title{
NEUROPATHOLOGICAL FINDINGS IN A CASE OF JUVENILE GENERAL PARESIS TREATED WITH PENICILLIN*
}

\author{
BY \\ R. M. NORMAN, H. URICH, AND W. A. HEATON-WARD \\ Burden Neuropathological Laboratory, Frenchay Hospital, Bristol and Stoke Park Hospital, Stapleton, Bristol
}

Until the present time there have been only three neuropathological reports of juvenile general paretics treated with penicillin. The two patients described by Smith and Morais (1948) died shortly after they had completed their treatment and the cerebral inflammatory changes were still intense. In the case reported by Gianascol, Weickhardt, and Neumann (1954), however, the paretic process had been arrested and the residual lesions of the cerebral cortex were similar to those seen in acquired stationary paresis treated with penicillin (Bruetsch, 1949) or with malaria alone (Sträussler and Koskinas, 1925; Wilson, 1928; Jakob, 1929). In the patient described below a small dose of penicillin was sufficient to abolish the paretic pattern of the cerebrospinal fluid but it did not arrest the slowly progressive course of the illness. Examination of the brain showed old lesions in the cerebellum, striatum, and ependyma consistent with the clinical diagnosis of juvenile paresis, but the changes in the cerebral cortex were surprisingly slight and were those of vascular rather than parenchymatous syphilis.

\section{Case Report}

A female child born on February 8, 1933, died on May 21, 1957.

Clinical History.-This girl, whose father died of general paresis, was apparently normal until the age of 8 years when she started deteriorating mentally. She continued, however, to attend an elementary school till the age of 11 years. At the age of 12 years she became a voluntary patient in a mental hospital, where she was found to be mentally subnormal (I.Q.57), and to need much supervision. The blood Kahn test was strongly positive and she received a course of Kharsulphan and Bismostab injections. The next year she was transferred to a mental deficiency hospital; at the examination her I.Q. was then 41 . There were no stigmata of congenital syphilis. She was subject to outbursts of temper and clothes tearing, unsteady in walking, and often incontinent. After 6 months she was reported to be strange

\footnotetext{
* Received for publication March 18, 1959.
}

in manner and was admitted to the colony hospital for observation. She was elated and excited, chattered incessantly with slurred articulation, and needed sedatives at night. She appeared to be suffering from delusions and talked to the staff about imaginary visits and numerous presents from her mother.

Ophthalmological examination showed a slight convergent strabismus. The visual fields and acuity were normal. The left pupil was irregular in outline and larger than the right, and both pupils reacted to light but not to accommodation.

The tongue on protrusion showed a coarse tremor. Muscle tone was reduced in the limbs, with poor coordination and diminished power. There was gross tremor in the hands when the arms were extended and dysdiadochokinesia was marked. Rombergism was present and the gait ataxic. There was a doubtful extensor plantar response on both sides.

Laboratory tests yielded the following results:

Blood: Wassermann reaction +++ ;

Cerebrospinal Fluid: cells 20 per c.mm. (mainly lymphocytes), protein $65 \mathrm{mg}$. per cent., globulin ++ , Lange 5555554210, Kahn test ++ .

A course of soluble penicillin was given by 3-hourly injections. The total dose was $1,600,000$ units.

A year later the cerebrospinal fluid showed: cells 1 per c.mm., protein $30 \mathrm{mg}$. per cent., globulin slightly increased, Lange 012222100 , Wassermann reaction + .

This striking change was not reflected in her clinical condition which failed to improve. Both her physical condition and the cerebrospinal fluid findings remained more or less stationary for the next 4 years. In 1952 she started having major epileptic fits which recurred at frequent intervals and were only partially controlled with barbiturates. In view of the repeatedly positive or doubtfully positive blood Kahn tests she was given another course of penicillin, consisting of injections of 300,000 units Procaine penicillin twice daily for 12 days. A month later the blood Kahn test was negative.

In 1954 the Wassermann reaction was negative in the cerebrospinal fluid but the Lange curve was 4332110000 .

Despite treatment the patient failed to improve, continued to have epileptic fits, became bedridden, developed pressure sores, and died of intercurrent infection at the age of 24 years. 


\section{Post mortem Findings}

Apart from bilateral hydronephrosis and acute pyelonephritis, the relevant findings were confined to the nervous system.

\section{Examination of the Central Nervous System}

Macroscopic.-The fixed brain weighed $1,124 \mathrm{~g}$. of which the cerebellum and brain stem accounted for $166 \mathrm{~g}$. The leptomeninges were slightly thickened and opaque, particularly over the superior frontal gyrus. They were not adherent and stripped easily without damage to the cortex. The convolutional pattern was normal and there was no obvious gyral atrophy. Coronal sections revealed the presence of numerous small gritty concrements in the anterior part of each putamen. Otherwise the pattern of grey and white matter appeared normal. The ventricles were not dilated. The cerebellum and brain stem showed no obvious lesions. The blood vessels and cranial nerves were normal.

Microscopic.-Large celloidin and frozen sections were prepared from representative parts of the cerebral cortex, basal ganglia, cerebellum, and brain stem, and stained with standard methods for cells, myelin, and glial fibres. In addition, selected sections were stained by the Prussian blue method for iron, Achúcarro-Klarfeld's method for reticulin fibres, and Cajal's silver nitrate-pyridine method for the cerebellum.
Meninges.-A mild degree of thickening and fibrosis was seen but no inflammatory infiltration. In some places dense aggregations of melanophores could be seen. The pial blood vessels were normal.

Cerebral Cortex.-On the convexity of the right occipital lobe there were two separate areas sharply distinguishable from the normal cortex and characterized by a loss of nerve cells and a greatly increased cellularity due to proliferation of astrocytes and microglia. Serial sections showed that these lesions varied in extent from a limited perivascular loss of nerve cells to a diffuse destruction especially of the outer layers which might involve the crowns and walls of two adjacent gyri (Fig. 1). The astrocytes in these areas showed active proliferation with swollen bodies and sometimes the formation of large multinucleated forms. Laminar proliferation of the smaller blood vessels with thickened adventitia and swollen endothelium was seen in areas of maximal nerve cell loss (Fig. 2, opposite). Neither the microglial cells nor the blood vessels contained stainable iron. In one of these occipital foci inflammatory round cells were scattered throughout the lesion and had aggregated into a dense nodule, about $0.2 \mathrm{~mm}$. in diameter, or irregular outline and devoid of any organized pattern (Fig. 3, opposite). The blood vessels at this site showed proliferated reticulin fibres (Fig. 4, opposite).

Clear-cut lesions of this type were not found in any other part of the brain. As regards the rest of the cerebral cortex it may be said that, in gereral, the nerve cells stained less deeply and therefore often appeared somewhat smaller than those of the normal adult. In many places the finer architectonic features of a normal cortex were somewhat blurred and the

FIG. 1.-Occipital cortex, showing intense gliosis and almost total loss of nerve cells in supragranular layers. Carbol azure $\times 55$. 


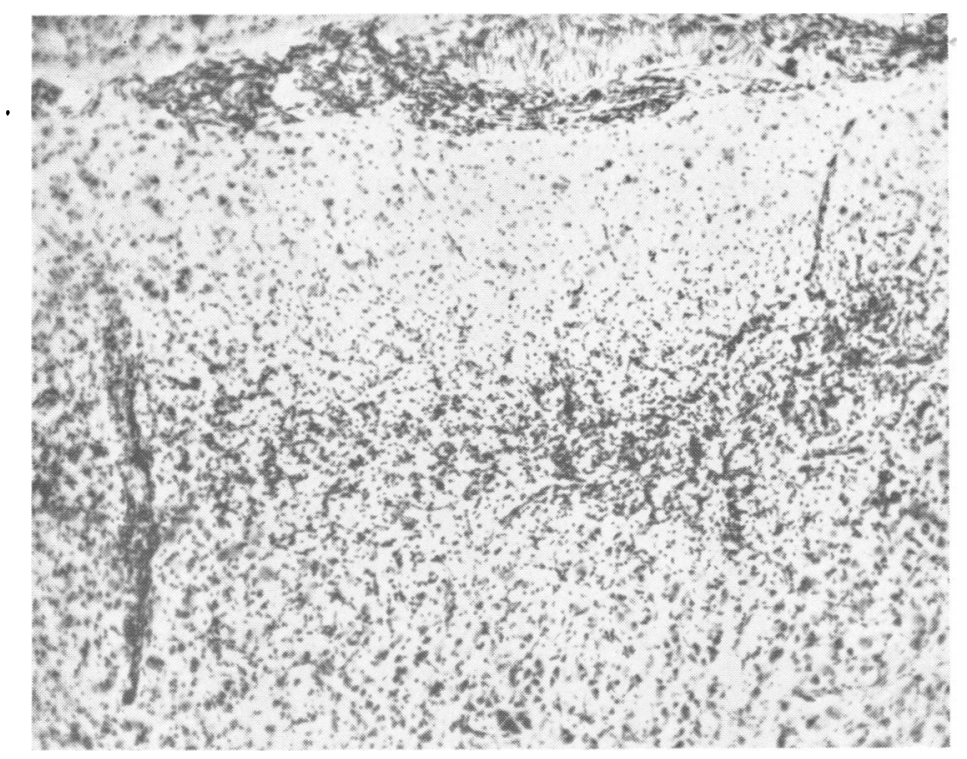

FIG. 2.-Occipital cortex, showing laminar thickening and proliferation of vessels. Numerous swollen astrocytes are present.

Carbol azure 80 .

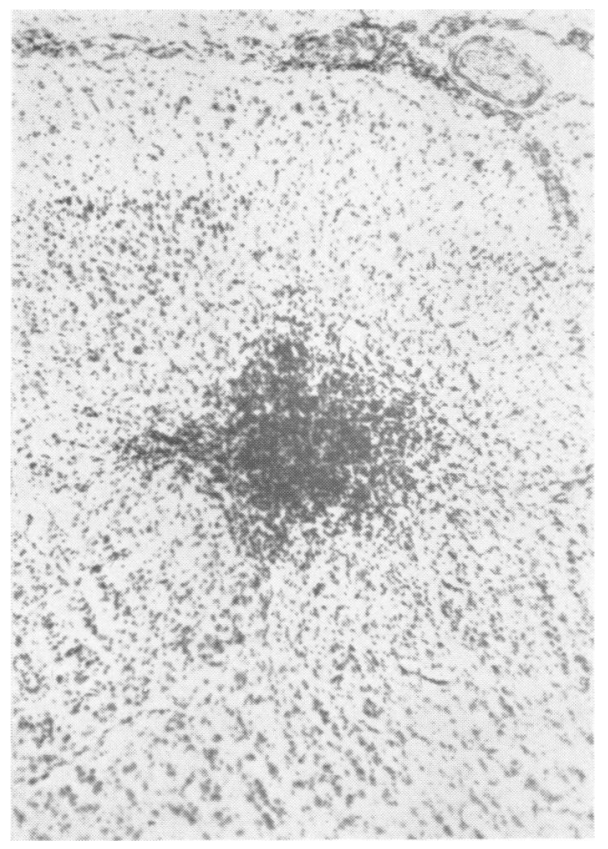

Fig. 3.-Occipital cortex, showing nodule of inflammatory
cells.

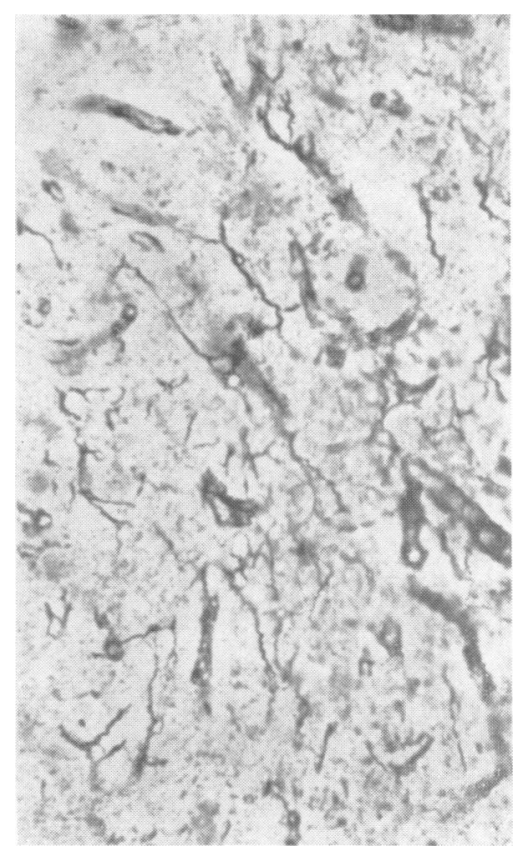

Fig. 4.-Proliferated reticulin fibres in the site of the nodule. 
linear arrangement of rows of nerve cells was less incisive (Fig. $5 a$ and $b$ ). A reduction in the number of nerve cells, however, could rarely be demonstrated (Fig. 6a and $b$ ) and when present was not accompanied by an active gliosis as seen in the occipital lobe. There was no gliosis or reduction in the intensity of myelin staining in the white matter and
Kultschitsky-Pal staining of frozen sections from different parts of the cortex revealed no detectable differences from the average normal. A small area of droplet calcification of the capillaries was found in the depths of the inferior temporal sulcus of the right side. Both hippocampal formations appeared normal.

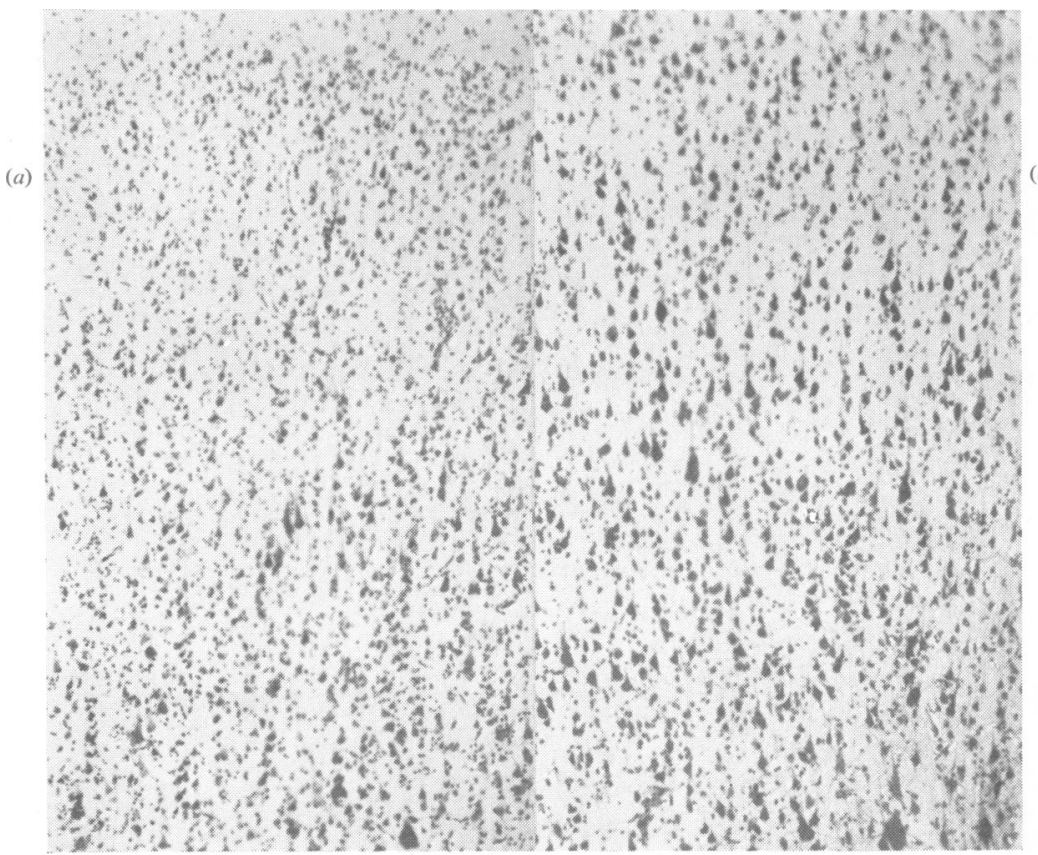

FIG. 5(a).-Outer layers of precentral gyrus, showing small size and poor arrangement of nerve cells.

Carbol azure $\times 60$.

FIG. 5(b).-As in Fig. 5(a), normal adult cortex.

Fig. 6(a).--Inferior parietal lobule, showing loss of nerve cells in third layer. Carbol azure $\times 60$.

FiG. 6(b).-As in Fig 6(a), normal adult cortex.

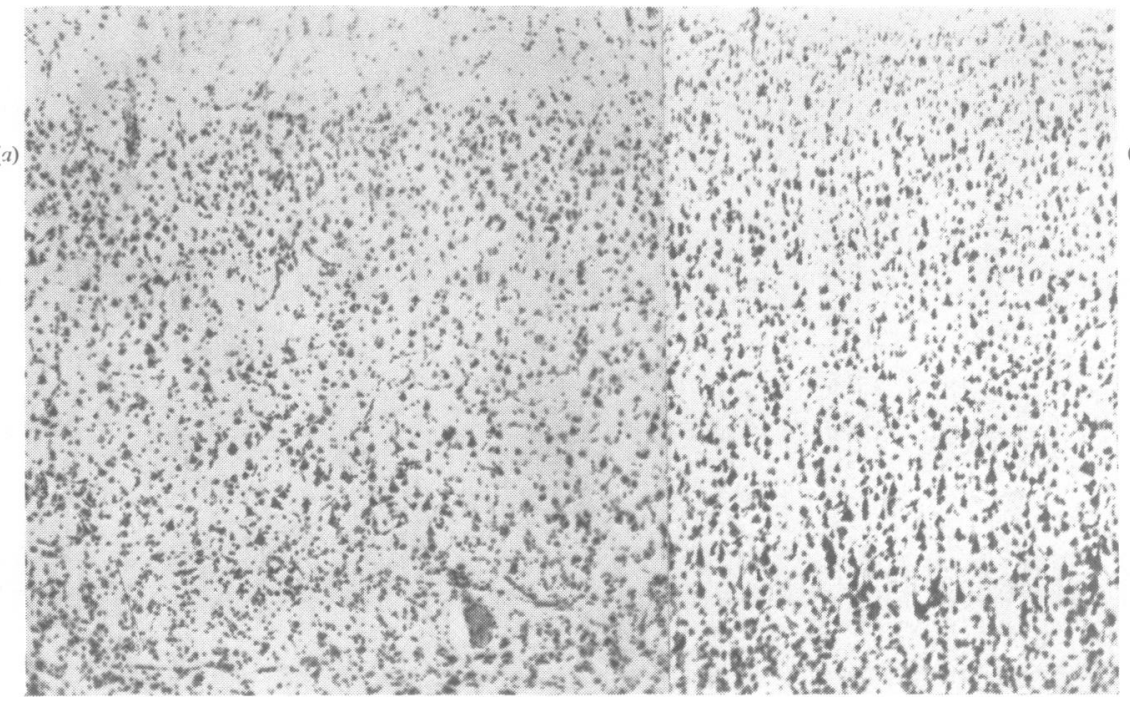


Basal Ganglia.-The only abnormality found was the presence of perivascular calcifications. They were mostly of pericapillary distribution and ranged from tiny droplets arranged along the capillary vessels to solid confluent concrements. Adventitial calcifications were also present in the walls of larger vessels, both arterial and venous. The calcifications were most numerous and dense in the anterior parts of each putamen at the level of the head of the caudate nucleus (Fig. 7). They were less dense in the central part of the putamen where they encroached upon the adjoining part of the external nucleus of the globus pallidus. Elsewhere in the pallidum, calcium deposits were confined to the adventitia of the larger vessels. Other parts of the basal ganglia showed no abnormality.

Brain Stem.-Apart from fibrillary gliosis in the marginal and subependymal zones and in the hila of the inferior olives, no abnormality could be seen.

Cerebellum.-The tips of several folia showed a moderate degree of atrophy with loss of Purkinje cells, rarefaction of the granular layer, and gliosis in the molecular layer (Figs 8 and 9).

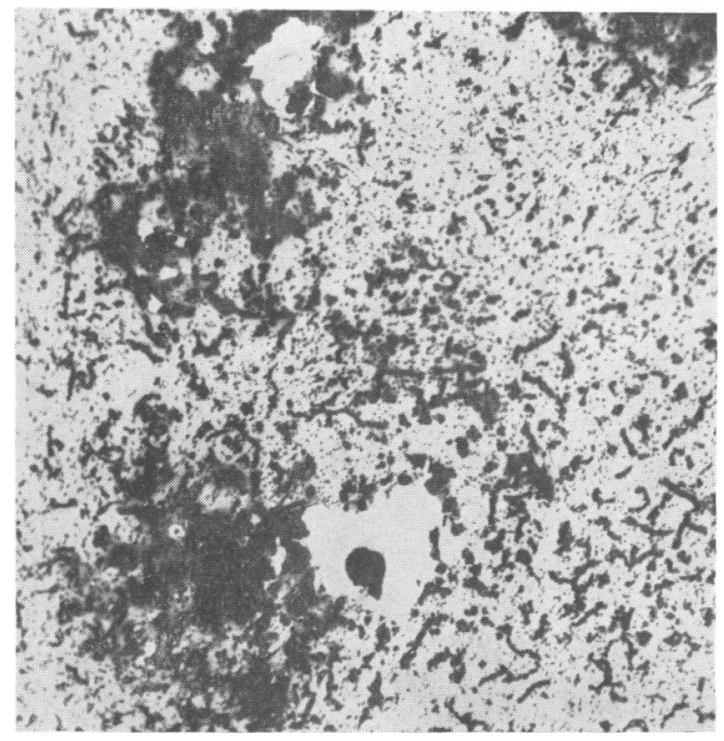

FIG. 7.-Putamen, showing perivascular and confluent calcifications. Carbol azure $\times 40$.

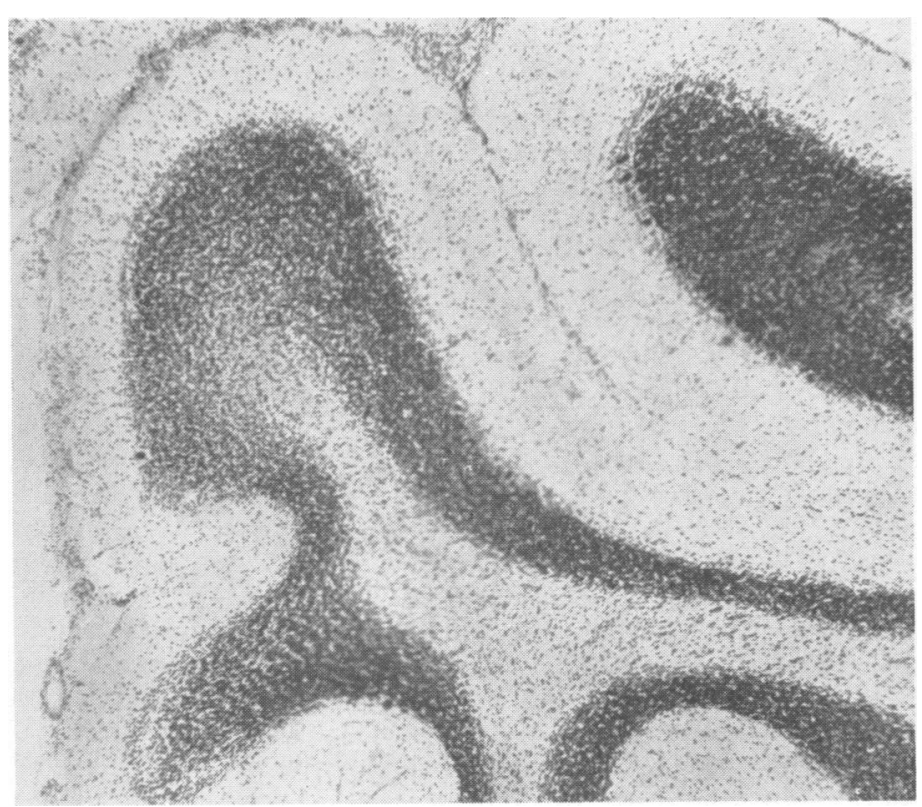

FIG. 8.-Cerebellum, showing loss of Purkinje cells and rarefaction of granule cells in two folia.
Carbol azure $\times 35$.

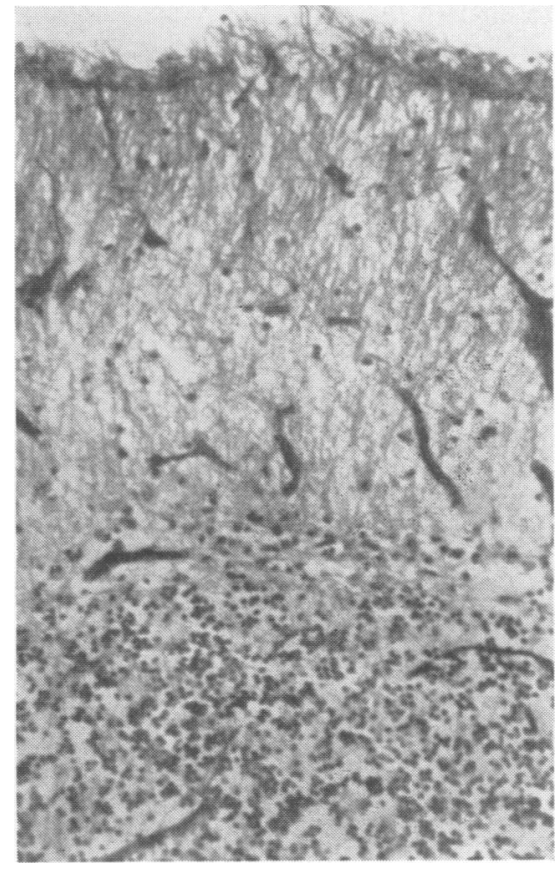

FIG. 9.-Cerebellum, showing gliosis in molecular layer of an atrophied folium. Holzer $\times 175$. 
In addition, a number of binucleated Purkinje cells were seen in various parts of the cerebellar cortex (Fig. 10).

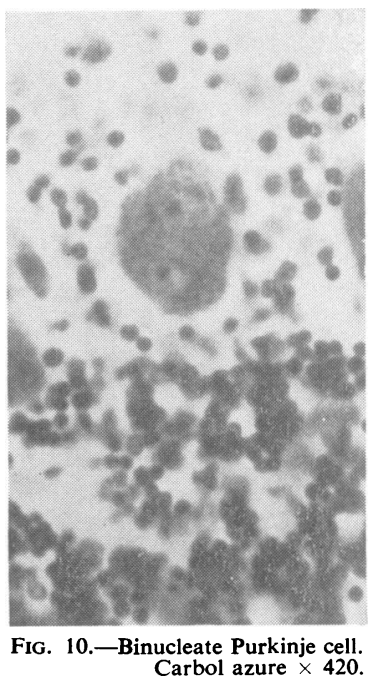

Degenerative changes in the Purkinje cells were very rare, only a few cells showing fusiform swellings of the axons. No dendritic expansions were seen. The cerebellar white matter was well myelinated and showed only a mild fibrillary gliosis. The dentate nuclei were normal.

Ependyma of the lateral and third ventricles showed a few small areas of denudation. A moderate subependymal gliosis was, however, present throughout the ventricular system. In the aqueduct and in the floor of the fourth ventricle, the changes were more pronounced and a mild granular ependymitis was present.

Choroid plexuses were normal.

Spinal cord showed no apparent lesions.

\section{Discussion}

The clinical diagnosis of juvenile general paresis is often applied to two distinct syndromes (Stewart, 1933). In one the child develops normally up to about the age of 10 years when an insidious and slowly progressive dementia sets in. In the other the child is mentally defective from birth, but may show signs of progressive deterioration in later childhood. The former type probably always represents true general paresis in which the pathological lesions closely resemble those found in the classical acquired disease. In the latter type these lesions are not necessarily found, and many cases are examples of primary mental deficiency, sometimes complicated by meningo-vascular syphilis. The differential diagnosis may be rendered difficult by the fact that changes in the cerebrospinal fluid of paretic type may be encountered in all forms of congenital syphilis of the central nervous system. Clinically, the present case probably belongs to the first group as the history suggests normal development up to the age of 8 years. From the pathological standpoint the residual lesions taken in combination offer strong support to the diagnosis of juvenile general paresis. The cerebellar changes are particularly important.

Atrophy of the cerebellar cortex, with predilection for the tips of the folia, first described by Sträussler (1910), is an almost invariable finding in juvenile paresis (Stewart, 1933). It has, however, also been reported in meningo-vascular syphilis and in suppurative meningitis, particularly in childhood (Noetzel, 1944).

Binucleated Purkinje cells, when first described by Sträussler (1906), were considered pathognomonic of juvenile paresis and were interpreted as a malformation indicative of the prenatal onset of the disease. Evidence has since accumulated in favour of their occasional occurrence in other diseases, some of which are undoubtedly acquired (Jakob, 1929). They are therefore considered to be a reactive phenomenon of limited specificity. Nevertheless, their presence in large numbers in an individual known to be suffering from congenital syphilis is strong supportive evidence in favour of true juvenile paresis.

The meningeal fibrosis, subependymal gliosis, and granular ependymitis, though typical features of general paresis, may also appear in other inflammatory diseases of the nervous system including meningo-vascular syphilis.

No specificity can be ascribed to aggregations of melanophores in the leptomeninges although this finding has been noted in adult general paresis (Jakob, 1929).

Perivascular calcifications in the striatum have previously been found in juvenile paresis by Roger, Alliez, and Gastaut (1948) and by van Bogaert (1949); both cases were of the Lissauer type. Calcifications are also mentioned in two older reports on acquired general paresis (Lubimoff, 1873; Ostertag, 1930), but details of the distribution are lacking.

A peculiar feature of the present case is the absence of old lesions in the cerebral cortex, particularly in view of the fact that widespread neuronal devastation is usual in juvenile paresis. The same problem arises in accounting for the remarkable 
"restitution" of the cortex in adult paretics successfully treated by malaria and/or penicillin. This wellattested finding is difficult to reconcile with Jakob's statement that, in all cases of general paresis, even in its earliest stages, extensive degeneration and loss of nerve cells are invariably found. The matter was put in a clearer light by Greenfield (1958), who pointed out that many cases of general paralysis coming to autopsy in an early stage of the disease show no cerebral abnormalities except for slight opacity of the leptomeninges and a few ependymal granulations. "Disagreement as to the effects of malarial treatment is due, no doubt, to the great variation in the intensity of these lesions before treatment was begun". Spielmeyer (1925) found no correlation between the lesions and the clinical course and pattern of general paresis, and considered that the inflammatory and degenerative processes are independent. Kral and Dörken (1953) also suggest that "at least two pathogenetic factors have to be considered: one probably connected with the inflammatory component and responsible for the acute clinical picture and the pathological changes in the cerebrospinal fluid and relatively easily influenced by our modern therapeutic methods; and another one, long acting, not easily or not at all influenced by therapy and responsible for the slowly progressive deterioration even in the therapeutically induced absence of pathological changes in the spinal fluid. There are reasons to believe that this second factor is a vascular one, as suggested by Merritt, Putnam, and Campbell (1937)".

A vascular lesion of this type was, in fact, present in the occipital lobe of our case where the NisslAlzheimer changes in the small intracortical vessels were demonstrated, but the neuronal destruction was of limited extent and could hardly have been responsible for the progressive clinical deterioration the cause of which remains obscure. The persistence, or appearance, of this vascular disease has been noted in some cases in which the paretic process had been arrested with malarial treatment (Gurewitsch, 1926; Wilson, 1928). The lesion in the present case was undoubtedly active, as shown by progressive changes in the astrocytes, by microglial activity, and by inflammatory round cell infiltration with the formation of a so-called "unorganized granuloma" (Jakob, 1929).

The remarkable and sustained change in the composition of the cerebrospinal fluid suggests that the original small dose of less than 2 mega units penicillin was sufficient to arrest the paretic process, but even the subsequent dose of $7 \cdot 2$ mega units failed to eradicate the infection. This view is supported by the observation of Bruetsch, who found a stainable spirochaete in the brain of an adult paretic apparently successfully treated with 6 mega units penicillin. Whether the modern dosage of 10 to 12 mega units will overcome this difficulty remains to be seen.

\section{Summary}

A girl of syphilitic parents developed symptoms of juvenile general paresis at the age of 8 years. She was treated with two courses of penicillin, one of 1.6 mega units, which abolished the paretic pattern of the cerebrospinal fluid, and 5 years later one of 7.2 mega units, which resulted in reversion of the serological reactions. Neither had any beneficial effect on her clinical condition, however, and she died at the age of 24 years.

Post mortem examination of the central nervous system revealed no signs of active general paresis in the cerebral cortex, the architecture of which was remarkably well preserved. Residual changes included slight meningeal thickening, granular ependymitis, mild cerebellar atrophy, presence of binucleated Purkinje cells, and calcifications in the basal ganglia. A small active lesion corresponding in type to the intracortical vascular syphilis of Nissl and Alzheimer was present in one occipital lobe. The presence of this lesion suggests that a dose of penicillin sufficient to arrest the paretic process is not necessarily adequate for complete eradication of the infection.

This work was carried out with the assistance of the Nuffield Foundation

\section{REFERENCES}

Bogaert, L. van, (1949). Mschr. Psychiat. Neurol., 118, 30.

Bruetsch, W. L. (1949). Dis. nerv. Syst., 10, 368.

Gianascol, A. J., Weickhardt, G. D., and Neumann, M.A. (1954). Amer. J. Syph., 38, 251.

Amer. J. Syph., 38, 251.
Greenfield, J. G. (1958). "Neuropathology", p. 167. Arnold, London.

Greenfield, J. G. (1958). "Neuropathology", p. 167. Arnold,

Jakob, A. (1929). "Anatomie und Histologie des Grosshirns", vol. 2, pt. 1 , pp. 603 et seq. Deuticke, Leipzig.

Kral, V. A., and Dörken, H. (1953). Amer. J. Psychiat.,109, 684.

Lubimoff, A. (1873). Virchows Arch. path. Anat., 57, 371.

Merritt, H. H., Putnam, T. J., and Campbell, A. C. P. (1937). Arch. Neurol.'Psychiat (Chicago), 37, 75.

Noetzel, H. (1944). Arch. Psychiat. Nervenkr., 117, 275.

Noetzel, H. (1944). Arch. Psychiat. Nervenkr., 117, 275.
Ostertag, B. (1930). Virchows Arch. path. Anat., 275, 828.

Ostertag, B. (1930). Virchows Arch. path. Anat., 275, 828.

Roger, H., Alliez, J., and Gastaut, H. (1948). Rev. Neurol., 80,
Smith, R. H. F., and Morais, V. de (1948). J. ment. Sci., 94, 70.

Spielmeyer, W. (1925). Z. ges. Neurol. Psychiat., 97, 287.

Stewart, R. M. (1933). J. ment. Sci., 79, 602.

Sträussler, E. (1906). Jb. Psychiat. Neurol., 27, 87

- (1910). Z. ges. Neurol. Psychiat., 2, 30.

and Koskinas, G. (1925). Ibid., 97, 176.

Wilson, R. B. (1928). Brain, 51, 440. 